\title{
Gene expression of ATP-binding cassette transporters during liver regeneration after $90 \%$ hepatectomy in rats
}

\author{
NORIHISA KIMURA, KENICHI HAKAMADA, SHOJIRO-KAZUNORI IKENAGA, \\ YUTAKA UMEHARA, YOSHIKAZU TOYOKI and MUTSUO SASAKI \\ Department of Gastroenterological Surgery, Hirosaki University Graduate School of Medicine, \\ Hirosaki 036-8562, Aomori Province, Japan
}

Received January 23, 2012; Accepted March 19, 2012

DOI: $10.3892 /$ ijmm.2012.972

\begin{abstract}
Liver damage with hyperbilirubinemia during regeneration of the small liver is the major hurdle to expand the indications of adult living donor liver transplantation. We performed a large-scale gene expression analysis of the regenerating liver after a $90 \%$ hepatectomy in rats, and analyzed the changes in the gene expression patterns related to the ATP-binding cassette (ABC) transporters. RNAs were prepared from 3 rat livers at 0, 24, 72 and $168 \mathrm{~h}$ after a $90 \%$ hepatectomy. The gene expression profile was analyzed by the Rat Genome 2302.0 array with special references to the ABC transporters. Among 31,042 probes, 1,587 reported genes were identified as either upregulated or downregulated more than 2-fold. Among 20 ABC transporter genes, multidrug resistance protein (MRP) 2 and organic anion transporting polypeptide (OATP) 1 were significantly downregulated, while MRP1 and MRP3 tended to be expressed. These genetic changes were confirmed by real-time PCR. A microarray analysis demonstrated not only an extensive gene expression profile in the regenerating liver but more specific molecular events related to bilirubin transport at the same time. Changes in the expression pattern of the ABC transporters, therefore, seem to be the key event in liver failure during liver regeneration.
\end{abstract}

Correspondence to: Dr Norihisa Kimura, Department of Gastroenterological Surgery, Hirosaki University Graduate School of Medicine, Hirosaki 036-8562, Aomori Province, Japan

E-mail: norihisa@cc.hirosaki-u.ac.jp

Abbreviations: SFSS, small-for-size graft syndrome; ABC transporter, ATP-binding cassette transporter; MRP, multidrug resistance protein; OATP, organic anion transporting polypeptide; NTCP, $\mathrm{Na}^{+}$-taurocholate cotransporting polypeptide; MDR, multidrug resistance P-glycoprotein; BSEP, canalicular bile salt export pump; $\mathrm{BCRP}$, breast cancer resistance protein; GSH transporter, glutathione transporter; GAPDH, glyceraldehyde-3-phosphate dehydrogenase

Keywords: small-for-size graft syndrome, massive hepatectomy, liver failure during liver regeneration, ATP-binding cassette transporter, multidrug resistance protein

\section{Introduction}

Post-operative liver damage with prolonged hyperbilirubinemia during liver regeneration is one of the major hurdles to expand the indications for liver surgery. It occurs mostly in patients receiving excess hepatectomy for various degrees of diseased livers, but also in recipients after a living-donor liver transplantation using small-for-size grafts of the normal liver (1-4). The latter is now called small-for-size graft syndrome (SFSS) (5).

SFSS usually occurs when the graft size is $<40 \%$ of the recipient's standard liver volume (4). Patients show marked cholestasis and various degrees of portal hypertension. Some patients recover spontaneously within a few months, but most require prolonged intensive care and the mortality rate is higher in comparison to larger grafts (3). Many hypotheses have been proposed regarding the mechanism of SFSS: shear stress injury by the excess portal flow into the relatively small liver $(6,7)$, free radical injury (8), and an imbalance of endothelin and intrinsic nitric oxide production (9). However, its details still remain unknown.

Regarding the mechanism of cholestasis in SFSS, an excretory step of bilirubin is thought to be impaired, because cholestasis is recognized as conjugated hyperbilirubinemia without biliary obstruction. Physiologically, conjugated bilirubin is evacuated from the cell into the bile canaliculi by a bilirubin-excreting transporter, an ATP-binding cassette (ABC) transporter called multidrug resistance protein (MRP) 2, which localizes to the canalicular membrane of the hepatocytes (10-12). In some pathological conditions, such as bile duct ligation (13-15), or under an endotoxin load (15-19), however, the expression of MRP2 is reported to be downregulated. As for liver regeneration, we previously reported that the MRP2 protein decreased and that MRP3, an isoform of MRP2, is expressed on the sinusoidal membrane of the hepatocytes, which leads to changes in the direction of the bilirubin transport from the bile canaliculi to the sinusoidal space (20). However, the genetic control of the bilirubin transporters during liver regeneration and the role of the other $\mathrm{ABC}$ transporters remain unclear.

A large number of genes participate in liver regeneration and various genes are expressed in the different phases. Given the recent development of a large-scale gene expression analysis by DNA microarray, it is now possible to thoroughly evaluate how many genes participate in liver regeneration 
$(21,22)$. Moreover, microarray technology allows us to analyze the chronological expression patterns of the genes with more specific functions at the same time.

In this study, we performed a large-scale gene expression analysis of the regenerating liver after a $90 \%$ hepatectomy in rats, then, analyzed the changes in the gene expression patterns related to the $\mathrm{ABC}$ transporters.

\section{Materials and methods}

Animals. Male Sprague-Dawley rats weighing 180-220 g and aged 6 wk were purchased from Charles River Laboratories Japan, Inc. (Tsukuba, Japan). They were kept under routine laboratory conditions at the animal laboratory of Hirosaki University. The rats received standard laboratory chow, had free access to food and water and were kept in a thermostatically controlled room $\left(25^{\circ} \mathrm{C}\right)$ with a 12-h light-dark cycle. Before undergoing the surgical procedures, all rats were fasted for $24 \mathrm{~h}$. After the surgical procedures, they had free access to a $200 \mathrm{~g} / \mathrm{l}$ glucose solution for $24 \mathrm{~h}$ to avoid post-operative hypoglycemia after the massive hepatectomy (23). This study was carried out in accordance with the Guidelines for Animal Experimentation, Hirosaki University, and all of the animals received humane care according to the criteria outlined in the 'Guide for the care and use of laboratory animals' prepared by the National Academy of Sciences and published by the National Institutes of Health (NIH publication 86-23, revised 1985).

Operative procedures and sample preparation . Forty-eight rats underwent an operation under isoflurane anesthesia. The rats were divided into two groups, as follows: i) the sham operation group $(n=24)$; after a midline incision of the abdomen, the suspending ligaments of the liver were dissected as far as possible. The hepatic arteries and the portal veins were dissected and the abdomen was then closed. ii) the $90 \%$ partial hepatectomy group $(n=24)$; a $90 \%$ partial hepatectomy was performed according to a new technique described by Kubota et al (24), which was modified from the original procedure established by Higgins and Anderson (25). It was reported that all of the $90 \%$ hepatectomy rats survived using this technique $(17,26)$. Briefly, after a midline incision of the abdomen, Glisson's sheaths were ligated separately and each liver lobe was ligated separately 2 or 3 times near the inferior vena cava. With this method, the median lobe, the left lateral lobe and the right lateral lobe were all resected completely without constricting the inferior vena cava or causing any bleeding.

The overall post-operative survival rate of the rats was $100 \%$ in both the sham operation and $90 \%$ hepatectomy groups. At 4 time points, $0,24,72$ and $168 \mathrm{~h}$ after hepatectomy, the rats were sacrificed to obtain liver samples by exsanguinations with sufficient isoflurane anesthesia. The liver tissue specimens were snap-frozen in liquid nitrogen and stored at $-80^{\circ} \mathrm{C}$ for the microarray and real-time PCR analysis. Data at each time point were obtained from 6 animals.

RNA isolation. Total-RNA was extracted from a $500 \mathrm{mg}$ frozen liver sample with TRIzol reagent (Invitrogen Life Terchnologies, Carlsbad, CA) following the manufacturer's protocol. In brief, a $100 \mathrm{mg}$ liver sample was homogenized in $1 \mathrm{ml}$ of TRIzol reagent containing phenol and guanidinium isothiocyanate/cationic detergent, followed by phenolchloroform extraction and isopropyl alcohol precipitation. We confirmed the RNA quality using an Agilent 2100 Bioanalyzer (Agilent Technologies, Palo Alto, CA).

Microarray hybridization and scanning. Using a poly-dT primer incorporating a T7 promoter, double-stranded cDNA was synthesized from $10 \mu \mathrm{g}$ of total-RNA using a one-cycle cDNA synthesis kit (Affymetrix, Santa Clara, CA). The double-stranded cDNA was then purified by spin columns (Affymetrix). Subsequently, biotin-labeled cRNA was generated from the double-stranded cDNA template through in vitro transcription with T7 polymerase using 3'-amplification reagents for IVT labeling (Affymetrix). The biotinylated cRNA was purified using an IVT cRNA cleanup kit (Affymetrix). The biotinylated cRNA $(20 \mu \mathrm{g})$ was fragmented in $40 \mathrm{mmol}$ of Tris-acetate ( $\mathrm{pH} \mathrm{8.1),} 100 \mathrm{mmol}$ of KOAc, and $30 \mathrm{mmol}$ of $\mathrm{MgOAc}$ for $35 \mathrm{~min}$ at $94^{\circ} \mathrm{C}$ to an average size of 35-200 bases. The fragmented, biotinylated cRNA $(10 \mu \mathrm{g})$, along with the hybridization controls (Affymetrix), were hybridized to GeneChip rat genome 2302.0 array (Affymetrix) containing probes for 31,042 transcripts. The arrays were hybridized for $16 \mathrm{~h}$ at $45^{\circ} \mathrm{C}$ and $60 \mathrm{r} / \mathrm{min}$. After hybridization, the arrays were washed and stained according to the standard antibody amplification for eukaryotic targets protocol (Affymetrix). The stained GeneChip arrays were then scanned with the GeneChip Scanner 3000 (Affymetrix) and GeneChip operating software program, ver. 1.2 (Affymetrix).

Microarray data analysis. The signal intensities were quantified by the GeneChip operating software program, ver. 1.2 (Affymetrix). Subsequently, we normalized the obtained numerical data with classical liner regression techniques. The hybridization intensity data were converted into a presence/ absence call for each gene, and the changes in gene expression between the experiments were detected by a comparison analysis. In order to extract the significant genes, the data were further analyzed using the GeneSpring software program ver. 7.2 (Agilent Technologies) using the Affymetrix gene ID and natural logalism. We selected the genes whose ratios were $>2$ or $<0.5$, namely, representing a 2 -fold difference in expression level. Furthermore, we obtained the well annotated full-length genes as the reported genes from the RefSeq database of the National Center for Biotechnology Information (NCBI) (www.ncbi.nlm.nih.gov). In order to analyze the reported gene expression data, we performed a QT cluster analysis. Preceding the QT cluster analysis, we carried out hierarchical clustering to appraise the number of groups. The molecular functions of these genes were determined while referring to the Gene Ontology database (www.geneontology.org).

Quantitative real-time PCR. TaqMan probes and primers for MRP1 (assay ID: Rn00574093_m1), MRP2 (Rn00563231_ m1), MRP3 (Rn00589786_m1), OATP1 (Rn00755148_m1) and glyseraldehyde-3-phosphate dehydrogenase (GAPDH) (Rn99999916_s1) were pre-designed assays-on-demand gene expression products (Applied Biosystems, Foster City, CA). We synthesized the first-strand cDNA with $1 \mu \mathrm{g}$ of total-RNA using oligo $\mathrm{d}(\mathrm{T}) 16$ primers and TaqMan reverse transcription reagents (Applied Biosystems). 1x TaqMan universal master 
A

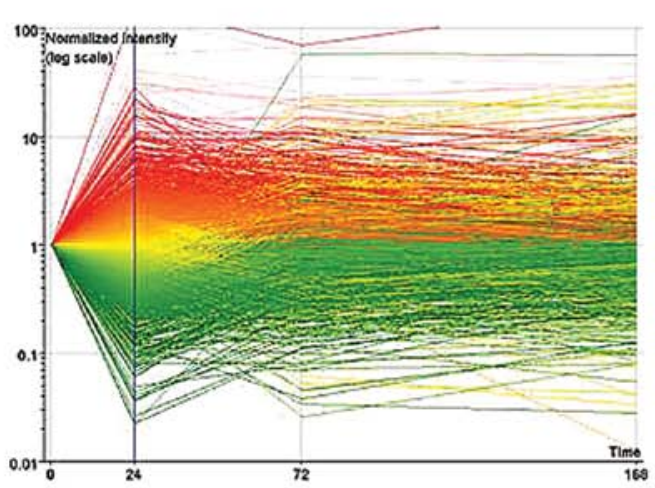

C

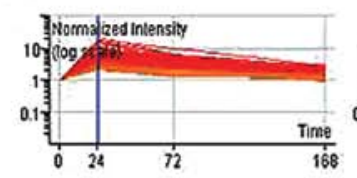

Set 1: 210 genes, 210 in list

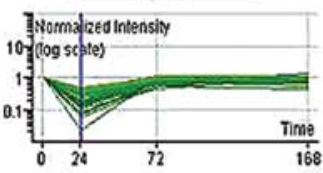

Set 5: 89 genes, 89 in list

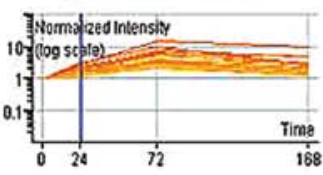

Set 9: 46 genes, 46 in list

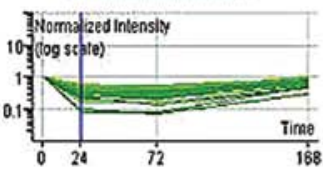

Sel 13: 38 genes, 38 in lis!

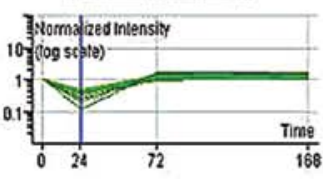

Sel 17: 27 genes, 27 in list

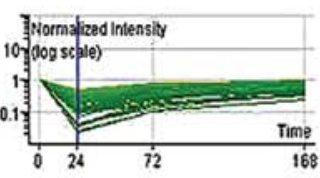

Set 2: 206 genes, 206 in list

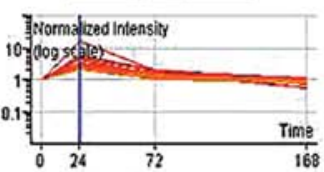

Sel 6:63 genes, 63 in list

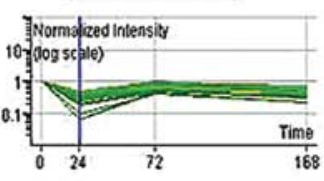

Set 10.42 genes, 42 in list

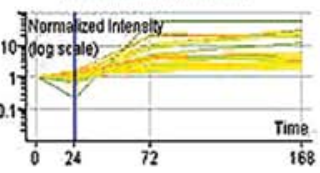

Sel 14: 36 genes, 36 in list

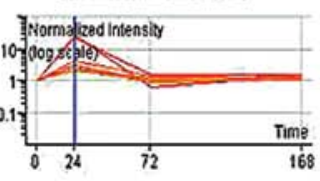

Set 18:23 genes, 23 ia list

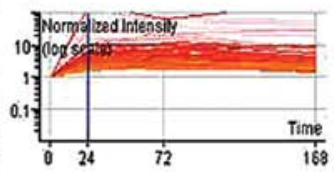

Sel $3: 157$ genes, 157 in list

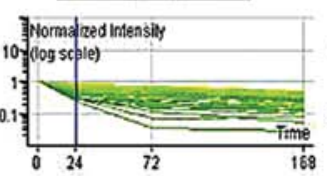

Sel 7: 54 genes, 54 in list:

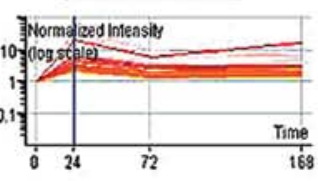

Sel11: 41 genes, 41 in list

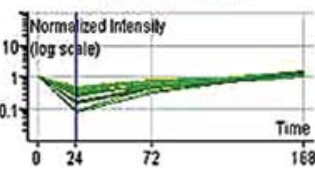

Se1 15: 33 genes. 33 in list

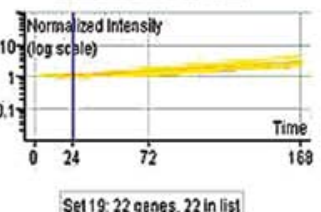

Set 19:22 genes, 22 in list

Yaxas Experiment Defaull interpretation

Solitby, OT clusters for I_z 2lovo (Defaual taterpretation)

Coloned br. Time 24

Gene List. IZYold (1587)

Figure 1. (A) Cluster of distribution trend. 1,587 reported genes differing with $>2$-fold intensity at least 1 time point during liver regeneration after a $90 \%$ hepatectomy were identified. (B) A hierarchical cluster analysis of 1,587 genes. A hierarchical cluster of 4 time points indicated that the genes in these time points hardly had a common expression profile. (C) A cluster analysis of differentially expressed genes identified by DNA microarray-based gene expression profiling. QT-clustering method was used and these genes were classified into 19 clusters.

mix (Applied Biosystems) was used as a PCR solution. TaqMan PCR was performed with an ABI PRISM 7700 sequence detector (Applied Biosystems) according to the manufacturer's instructions. All of the experiments were performed in duplicate and non-template controls, and dissociation curves were used to detect primer-dimer conformation and nonspecific amplification. The threshold cycle $\left(\mathrm{C}_{\mathrm{T}}\right)$ of each target product was determined and set in relation to the amplification plot of GAPDH. The detection threshold was set to the log linear range of the amplification curve and kept constant (0.05) for all of the data analysis. The difference in the $\mathrm{C}_{\mathrm{T}}$ values $\left(\Delta \mathrm{C}_{\mathrm{T}}\right)$ between the 2 genes was used to calculate the relative expression: relative expression $=2^{-\left(\mathrm{C}_{\mathrm{T}} \text { of target gene }-\mathrm{C}_{\mathrm{T}} \text { of } \mathrm{GAPDH}\right)}=2^{-\Delta \mathrm{C}_{\mathrm{T}}}$.

Statistical analysis. Data were expressed as the mean $\pm \mathrm{SD}, \mathrm{n}=6$, and Mann-Whitney U test (non-parametric test) was used to 
A

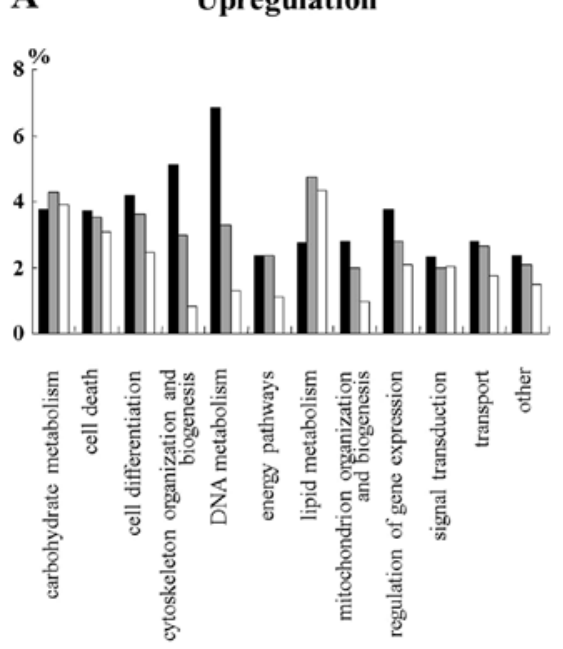

B
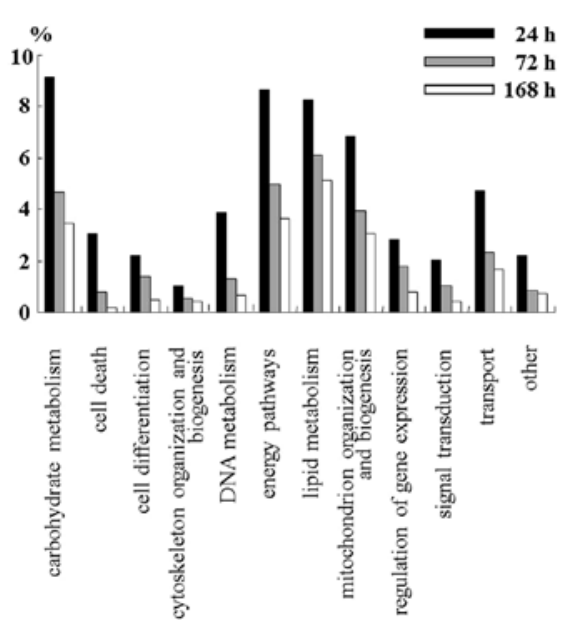

Figure 2. Percentage of changed genes according to the Gene Ontology classification. The genes were either (A) upregulated or (B) downregulated according to the molecular functions during liver regeneration.

determine the significance between the control (sham operation) and the $90 \%$ partial hepatectomy groups at 4 time points $(0,24$, 72 and $168 \mathrm{~h}$ ) for the expression of MRP1, MRP2, MRP3 and OATP1. $\mathrm{P}<0.05$ was considered to be statistically significant.

\section{Results}

Liver regeneration after a $90 \%$ partial hepatectomy. The wet liver weight showed a linear increase $(0.04 \mathrm{~g} / \mathrm{h})$ until $72 \mathrm{~h}$ after the $90 \%$ partial hepatectomy and the rate decreased thereafter. At $168 \mathrm{~h}$, the weight reached $60 \%$ (data not shown).

Metabolism of organic anions. We previously reported the changes in the total and direct bilirubin at 12,24,36, 48, 72 and $168 \mathrm{~h}$ after a $90 \%$ hepatectomy (20). The serum total bilirubin showed a rapid increase and reached a peak level of about $2 \mathrm{mg} / \mathrm{dl}$ at $36 \mathrm{~h}$ after the $90 \%$ hepatectomy, which returned to the preoperative level by $168 \mathrm{~h}$. There was a much greater increase in the serum direct bilirubin levels in comparison to the indirect bilirubin levels. In the sham operation group, there was little change in the total and direct bilirubin levels postoperatively (data not shown).

Application of the DNA microarray analysis for detection of changes in gene expression during liver regeneration. In order to comprehensively examine the sequential changes in gene expression during liver regeneration, we performed a DNA microarray analysis. As a result of the Affymetrix GeneChip analysis of the 31,042 probes, 4,722 genes were identified as up- or downregulated at least $>2$-fold at one time point during liver regeneration, of which 1,587 genes were reported as the well annotated full-length genes, and 3,135 were unreported genes (Fig. 1A).

Hierarchical cluster analysis of genes expressed during liver regeneration. A cluster analysis of the genes expressed at 4 time points $(0,24,72$ and $168 \mathrm{~h})$ during liver regeneration showed that the reported 1,587 genes altered at least $>2$-fold in intensity at 1 time point, and that the most similar patterns of gene expression were located next to each other and placed in a major branch of the dendrogram (Fig. 1B). In order to facilitate the visualization and interpretation of the gene expression program represented in this very large body of data, we used QT-clustering to order the genes on the basis of similarities in their expression patterns and displayed the results in a compact graphical format, generating 19 kinds of ramose gene expression clusters (Fig. 1C).

Percentage of changed genes according to the molecular functions determined by the gene ontology database. The genes were categorized into 12 groups based on their functions. The genes categorized to DNA metabolism, cytoskeleton organization and biogenesis, and cell differentiation tended to be upregulated in the earlier phase of liver regeneration (Fig. 2A), while those of carbohydrate metabolism, energy pathways, lipid metabolism, and mitochondrion organization and biogenesis were downregulated in the same phase (Fig. 2B).

Expression changes of $A B C$ transporter genes during liver regeneration by DNA microarray analysis. A subgroup of $20 \mathrm{ABC}$ transporter genes was extracted from the microarray data base and their serial changes were analyzed (Fig. 3A). As for OATPs and NTCP, the uptake transporters for bilirubin and bile acid on the sinusoidal membrane, the gene expressions of OATP1, OATP2, OATP4 and NTCP were strongly downregulated at $24 \mathrm{~h}$, but returned to normal by $168 \mathrm{~h}$ (Fig. 3B). In the MRP family, the MRP1 gene, one of the excreting transporters on the sinusoidal membrane, was upregulated $>2$-fold between 24 and $72 \mathrm{~h}$. In contrast, the gene levels of MRP2 (2-fold) and MRP6 (3-fold) were significantly downregulated at $24 \mathrm{~h}$ (Fig. 3C). In the other excreting transporters, the expression of the MDR $1 \mathrm{~b}$ gene was strongly upregulated $>5$-fold at $24 \mathrm{~h}$, but returned to normal by $168 \mathrm{hr}$. In contrast, the MDR1a gene level was significantly downregulated $>2$-fold at $24 \mathrm{~h}$, and MDR2 was significantly downregulated $>2$-fold at $168 \mathrm{~h}$. BCRP was significantly upregulated $>2$-fold at $72 \mathrm{~h}$ (Fig. 3D). 

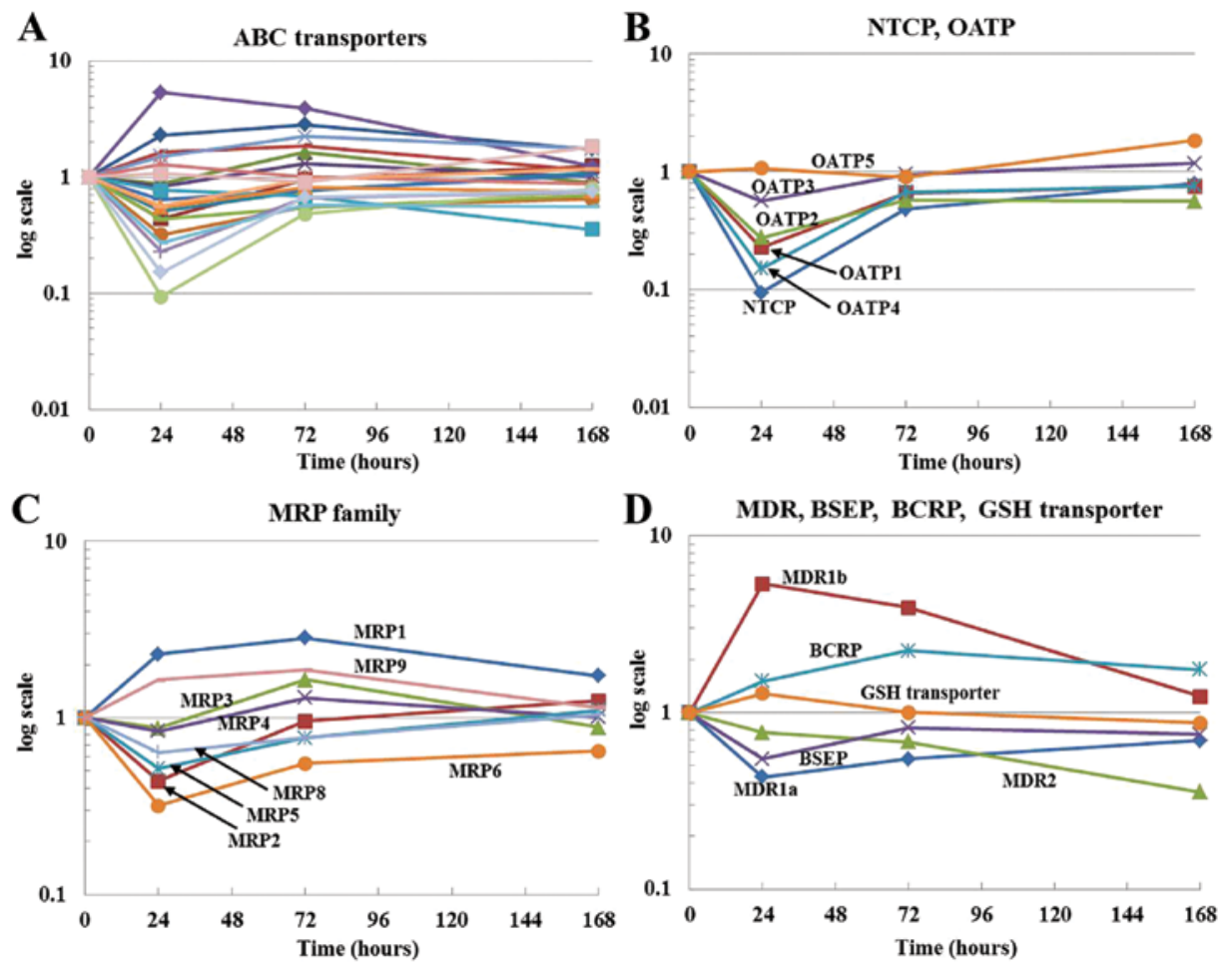

Figure 3. Microarray analysis of $\mathrm{ABC}$ transporter genes. (A) Data on the genetic expressions of $20 \mathrm{ABC}$ transporters were extracted from the database of microarray analyses. (B) As for the uptake transporters, the gene expressions of OATP1, OATP2, OATP4 and NTCP were strongly downregulated at $24 \mathrm{~h}$, but returned to normal by $168 \mathrm{~h}$. (C) The MRP1 gene was upregulated $>2$-fold between 24 and $72 \mathrm{~h}$, while the gene levels of MRP2 (2-fold) and MRP6 (3-fold) were significantly downregulated at $24 \mathrm{~h}$. (D) In the other excreting transporters, the expression of the MDR $1 \mathrm{~b}$ gene was strongly upregulated $>5$-fold at $24 \mathrm{~h}$, but returned to normal by $168 \mathrm{~h}$. In contrast, the MDR1a gene level was significantly downregulated $>2$-fold at $24 \mathrm{~h}$, and MDR2 was significantly downregulated $>2$-fold at $168 \mathrm{~h}$. BCRP was significantly upregulated $>2$-fold at $72 \mathrm{~h} .$.
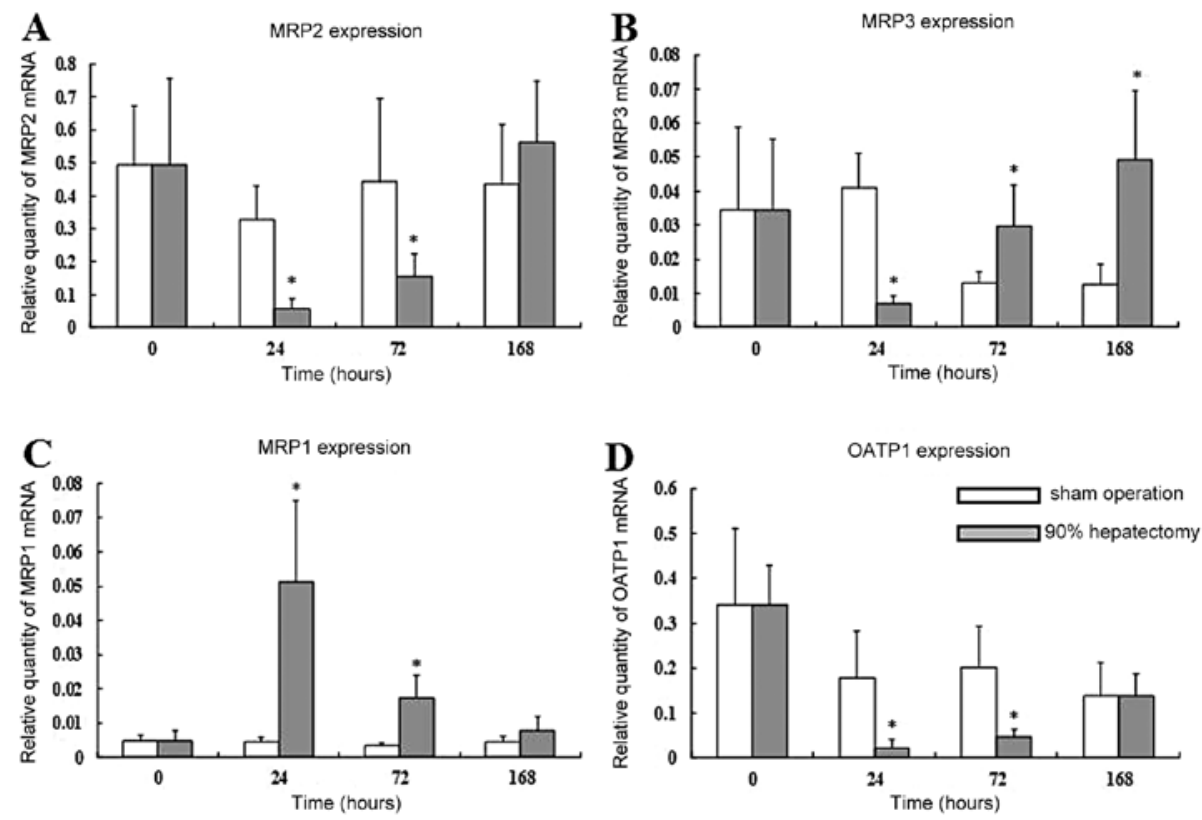

Figure 4. Real-time PCR analysis of ABC transporter genes. (A) MRP2; (B) MRP3; (C) MRP1; (D) OATP1, ABC transporters related to the bilirubin transportation by real-time PCR. MRP2 and OATP1 significantly decreased and MRP1 increased between 24 and $72 \mathrm{~h}$. MRP3 significantly decreased at $24 \mathrm{~h}$ and then significantly increased between 72 and $168 \mathrm{~h}$. ${ }^{*} \mathrm{P}<0.05$, in comparison to the sham operation.

$M R P 2, M R P 3, M R P 1$ and $O A T P 1$ gene expression by real-time $P C R$. In order to estimate the quantitative gene expression of the $\mathrm{ABC}$ transporters related to the bilirubin transportation, we selected four genes; MRP2, MRP3, MRP1 and OATP1 of the
OATPs. In the $90 \%$ hepatectomy group, both MRP2 and OATP1 significantly decreased and MRP1 increased after $24 \mathrm{~h}(\mathrm{P}<0.05)$ in comparison to those in the shame operation group. MRP3 significantly decreased at $24 \mathrm{~h}$, but increased after $72 \mathrm{~h}$ (Fig. 4). 


\section{Discussion}

Under specific circumstances, such as obstructive jaundice and sepsis, it has been reported that the expression of MRP2 on the canalicular membrane decreases and that MRP3 is expressed on the sinusoidal membrane. Consequently, the direction of bilirubin transport changes from toward the bile canaliculi to toward the sinusoidal space, which leads to conjugated hyperbilirubinemia $(13,14)$. As for the expression of the $\mathrm{ABC}$ transporters during liver regeneration of the small liver, we previously demonstrated the same patterns of MRP2 and MRP3 expression in rat hepatocytes by a western blot analysis (20). However, the changes in the genetic expression of these transporters have not yet been analyzed. Because many other ABC transporters have now been identified, a more comprehensive analysis of the expression of these genes is necessary (27-31). Moreover, with the development of cDNA microarray technology, we are allowed to analyze the whole genome expression in a specific condition semiquantitatively.

In the present study, we performed a DNA microarray analysis of the liver tissue samples first. Four measurement time points were adopted; $0,24,72$ and $168 \mathrm{~h}$. There are some reports of microarray analyses related to liver regeneration, but most have been measured in the early phases of regeneration (26,32-35). Because our concern is more specific to the changes in the $\mathrm{ABC}$ transporters, we analyzed the later phase according to the data obtained in the previous report (20). Consequently, among the 31,042 genes, 1,587 (5.1\%) of the reported genes were identified to be associated with rat liver regeneration after a $90 \%$ hepatectomy. Following the cluster analysis, 1,587 genes showed 19 expression profiles based on the similarity of the changed genes. Moreover, while referring to the Gene Ontology database, the genes were categorized into 12 groups based on their functions. Among them, the genes categorized to cell death, cell differentiation, cytoskeleton organization and biogenesis, and DNA metabolism tended to increase in the earlier phase of liver regeneration, while those of carbohydrate metabolism, energy pathways, lipid metabolism, mitochondrion organization and biogenesis tended to decrease in the same phase. Because these gene expression profiles seemed to meet the pattern of liver regeneration reported earlier $(36,37)$, we considered that our rat $90 \%$ hepatectomy model was therefore useful for further specific analyses of the ABC transporters during liver regeneration.

As for the ABC transporters, the gene expressions related to uptake of bilirubin (OATP) and bile acid (NTCP) into the hepatocytes were uniformly decreased during the observation periods. The expression of MRP2 was downregulated early after the hepatectomy, which supported our previous results of western blot analysis (20). MRP3, on the contrary, showed a slight increase. Indeed, the results seemed different from our previous study, which disclosed the apparent expression pattern of MRP3 after a rat $90 \%$ hepatectomy, but the level of MRP3 expression in normal livers is quite low, so that a tiny change in MRP3 expression may be overestimated by western blot analysis. On the other hand, MRP1, which was reported to be localized to the sinusoidal membrane and carry bilirubin toward the sinusoid similarly to MRP3, was expressed throughout the regeneration process.
These data obtained from microarray analyses were parallel to the results from real-time PCR. Real-time PCR disclosed the expressions of these genes related to bilirubin transport more clearly. The function of MRP1 and MRP3 was reported to be the same, but the time course of gene expression was different between the two genes. MRP3 probably plays more of a role in the later phase of regeneration than MRP1 does. Consequently, data from microarray analyses and realtime PCR disclosed that the gene expressions were changed to carry bilirubin from toward the bile canaliculi to toward the sinusoid, as we previously reported by western blot analysis. The gene expression profiles of the bile acid transports showed a different pattern from those of bilirubin.

We herein demonstrated a possible mechanism of conjugated hyperbilirubinemia during the regeneration of a small liver. The downregulation of MRP2 seems to be the direct cause of conjugated hyperbilirubinemia, and the regulation of this gene expression may lead to overcome cholestasis during regeneration of the small liver. However, the cascade of reactions leading to MRP2 downregulation remains unknown. In our previous paper, we mentioned a hypothesis that the increased plasma levels of interleukin 6 may play a role in the downregulation of the MRP2 protein during liver regeneration (20). Importance of activation of interleukin 6 and tumor necrosis factor during early phase of liver regeneration after partial hepatectomy has been reported: tumor necrosis factor binds the receptor on Kupffer cells, leading to the activation of nuclear factor kappa B. Interleukin 6 and tumor necrosis factor are both nuclear factor kappa B target genes, so that interleukin 6 is released into the serum and binds to the receptor on hepatocytes. Subsequently, a signaling cascade including phosphorylation of signal transducer and activator of transcription 3 and extracellular signal-regulated kinase $1 / 2$ leads the upregulation of multiple genes important for regeneration $(36,38)$. Regarding the effects of cytokines on the expression of MRP family, expression of MRP2 is reported to be downregulated in endotoxin-loading models, in which increases in liver mRNA and serum tumor necrosis factor and interleukin 6 are observed $(17,19,38)$.

In the present study, we analyzed the genetic expressions during the later phase of liver regeneration with special reference to the $\mathrm{ABC}$ transporters by both microarray and real-time PCR analyses. Indeed, real-time PCR is a more precise method for evaluating gene expression changes, but it is very difficult to apply real-time PCR for a comprehensive analysis of many complicated processes such as liver regeneration at the same time. From the improvement of microarray technology, data from the microarray analysis have become parallel to those from real-time PCR like our study. The comprehensive microarray analysis is thus considered to be a promising technology to increase the understanding of liver regeneration as a whole and more specific phenomena at the same time.

In summary, persistent elevation of serum bilirubin during the regeneration of a small liver was suggested to be due to sustained low uptake from the sinusoids by repressed OATP1 and low export form hepatocytes to bile duct by repressed MRP2 on the canalicular membrane. In addition to these regulations, the increased export from hepatocytes to blood by MRP1 and MRP3 on the basolateral membrane may be an important mechanism of persistent cholestasis during the regeneration of a small liver. 


\section{Acknowledgements}

The authors thank Dr Tung-Huei Chang for his various contributions throughout the study.

\section{References}

1. Emond JC, Renz JF, Ferrell LD, et al: Functional analysis of grafts from living donors. Implications for the treatment of older recipients. Ann Surg 224: 544-552, 1996.

2. Kawasaki S, Makuuchi M, Matsunami $\mathrm{H}$, et al: Living related liver transplantation in adults. Ann Surg 227: 269-274, 1998.

3. Kiuchi T, Kasahara M, Uryuhara K, et al: Impact of graft size mismatching on graft prognosis in liver transplantation from living donors. Transplantation 67: 321-327, 1999.

4. Sugawara Y, Makuuchi M, Takayama T, et al: Small-for-size grafts in living-related liver transplantation. J Am Coll Surg 192: 510-513, 2001.

5. Ben-Haim M, Emre S, Fishbein TM, et al: Critical graft size in adult-to-adult living donor liver transplantation: impact of the recipients disease. Liver Transpl 7: 948-953, 2001.

6. Oya H, Sato Y, Yamamoto S, et al: Surgical procedures for decompression of excessive shear stress in small-for-size living donor liver transplantation--new hepatic vein reconstruction. Transplant Proc 37: 1108-1111, 2005.

7. Takada Y, Ueda M, Ishikawa Y, et al: End-to-side portocaval shunting for a small-for-size graft in living donor liver transplantation. Liver Transpl 10: 807-810, 2004.

8. Zhong Z, Connor HD, Froh M, et al: Free radical-dependent dysfunction of small-for-size rat liver grafts: prevention by plant polyphenols. Gastroenterology 129: 652-664, 2005.

9. Palmes D, Minin E, Budny T, et al: The endothelin/nitric oxide balance determines small-for-size liver injury after reduced-size rat liver transplantation. Virchows Arch 447: 731-741, 2005.

10. Keppler D and Konig J: Hepatic secretion of conjugated drugs and endogenous substances. Semin Liver Dis 20: 265-272, 2000.

11. Konig J, Nies AT, Cui Y, Leier I and Keppler D: Conjugate export pumps of the multidrug resistance protein (MRP) family: localization, substrate specificity, and MRP2-mediated drug resistance. Biochim Biophys Acta 1461: 377-394, 1999.

12. Trauner M, Meier PJ and Boyer JL: Molecular pathogenesis of cholestasis. N Engl J Med 339: 1217-1227, 1998.

13. Donner MG and Keppler D: Up-regulation of basolateral multidrug resistance protein 3 (Mrp3) in cholestatic rat liver. Hepatology 34: 351-369, 2001.

14. Tanaka Y, Kobayashi Y, Gabazza EC, et al: Increased renal expression of bilirubin glucuronide transporters in a rat model of obstructive jaundice. Am J Physiol Gastrointest Liver Physiol 282: G656-G662, 2002.

15. Trauner M, Arrese M, Soroka CJ, et al: The rat canalicular conjugate export pump (Mrp2) is down-regulated in intrahepatic and obstructive cholestasis. Gastroenterology 113: 255-264, 1997.

16. Elferink MG, Olinga P, Draaisma AL, et al: LPS-induced downregulation of MRP2 and BSEP in human liver is due to a posttranscriptional process. Am J Physiol Gastrointest Liver Physiol 287: G1008-G1016, 2004.

17. Kubitz R, Wettstein M, Warskulat $U$ and Haussinger D: Regulation of the multidrug resistance protein 2 in the rat liver by lipopolysaccharide and dexamethasone. Gastroenterology 116: 401-410, 1999
18. Nakamura J, Nishida T, Hayashi K, et al: Kupffer cell-mediated down regulation of rat hepatic CMOAT/MRP2 gene expression. Biochem Biophys Res Commun 255: 143-149, 1999.

19. Vos TA, Hooiveld GJ, Koning H: Up-regulation of the multidrug resistance genes, Mrp1 and Mdrlb, and down-regulation of the organic anion transporter, Mrp2, and the bile salt transporter, Spgp, in endotoxemic rat liver. Hepatology 28: 1637-1644, 1998.

20. Chang TH, Hakamada K, Toyoki Y, Tsuchida S and Sasaki M: Expression of MRP2 and MRP3 during liver regeneration after 90\% partial hepatectomy in rats. Transplantation 77: 22-27, 2004.

21. Ning QJ, Qin SW and Xu CS: Expression patterns and action analysis of genes associated with drug-induced liver diseases during rat liver regeneration. World J Gastroenterol 12: 6966-6972, 2006.

22. Xu CS, Chang CF, Yuan JY, et al: Expressed genes in regenerating rat liver after partial hepatectomy. World J Gastroenterol 11: 2932-2940, 2005

23. Gaub J and Iversen J: Rat liver regeneration after $90 \%$ partial hepatectomy. Hepatology 4: 902-904, 1984.

24. Kubota T, Takabe K, Yang M, et al: Minimum sizes for remnant and transplanted livers in rats. J Hepatobiliary Pancreat Surg 4: 398-404, 1997.

25. Higgins $G$ and Anderson R: Experimental pathology of the liver. Arch Pahol 12: 186-202, 1931.

26. Morita T, Togo S, Kubota T, et al: Mechanism of postoperative liver failure after excessive hepatectomy investigated using a cDNA microarray. J Hepatobiliary Pancreat Surg 9: 352-359, 2002.

27. Jansen PL and Sturm E: Genetic cholestasis, causes and consequences for hepatobiliary transport. Liver Int 23: 315-322, 2003.

28. Kullak-Ublick GA, Stieger B and Meier PJ: Enterohepatic bile salt transporters in normal physiology and liver disease. Gastroenterology 126: 322-342, 2004.

29. Meier Y, Pauli-Magnus C, Zanger UM, et al: Interindividual variability of canalicular ATP-binding-cassette (ABC)-transporter expression in human liver. Hepatology 4: 62-74, 2006.

30. Ros JE, Roskams TA, Geuken M, et al: ATP binding cassette transporter gene expression in rat liver progenitor cells. Gut 52: 1060-1067, 2003

31. Trauner M and Boyer JL: Bile salt transporters: molecular characterization, function, and regulation. Physiol Rev 83: 633-671, 2003.

32. Arai M, Yokosuka O, Chiba T, et al: Gene expression profiling reveals the mechanism and pathophysiology of mouse liver regeneration. J Biol Chem 278: 29813-29818, 2003.

33. Dransfeld O, Gehrmann T, Kohrer K, et al: Oligonucleotide microarray analysis of differential transporter regulation in the regenerating rat liver. Liver Int 25: 1243-1258, 2005.

34. Locker J, Tian J, Carver R, et al: A common set of immediateearly response genes in liver regeneration and hyperplasia. Hepatology 38: 314-325, 2003.

35. Togo S, Makino H, Kobayashi T, et al: Mechanism of liver regeneration after partial hepatectomy using mouse cDNA microarray. J Hepatol 40: 464-471, 2004.

36. Fausto N, Campbell JS and Riehle KJ: Liver regeneration. Hepatology 43: S45-S53, 2006.

37. Michalopoulos GK and DeFrances MC: Liver regeneration. Science 276: 60-66, 1997

38. Huang W, Ma K, Zhang J, et al: Nuclear receptor-dependent bile acid signaling is required for normal liver regeneration. Science 312: 233-236, 2006. 